\title{
Complete androgen insensitivity syndrome and risk of gonadal malignancy: systematic review
}

\begin{abstract}
Beatriz Amstalden Barros ${ }^{1,2}$ Letícia Ribeiro de Oliveira ${ }^{3}$, Cíntia Regina Crocetti Surur', Antonio de Azevedo BarrosFilho², Andrea Trevas MacielGuerra $^{1,4}$, Gil Guerra-Junior ${ }^{1,2}$

IInterdisciplinary Group for the Study of Sex Determination and Differentiation (GIEDDS), State University of Campinas (UNICAMP), Campinas/São Paulo, Brazil ${ }^{2}$ Department of Pediatrics, State University of Campinas (UNICAMP), Campinas/São Paulo, Brazil

${ }^{3}$ Department of Pediatrics, Federal University of Uberlândia (UFU), Uberlândia/Minas Gerais, Brazil

${ }^{4}$ Department of Medical Genetics, State University of Campinas (UNICAMP), Campinas/São Paulo, Brazil
\end{abstract}

Complete androgen insensitivity syndrome (CAIS) is a rare condition characterized by $46, X Y$ karyotype, female external genitalia, absence of uterus, and testes located intra-abdominally, in the inguinal ring or in the labia majora. In the present study, the frequency of testicular malignancy in prepubertal and pubertal patients with CAIS who underwent gonadectomy or gonadal biopsy were evaluated. Systematic review was performed using electronic databases according to the PRISMA-P (Preferred Reporting Items for Systematic Review and Meta-Analysis Protocols) guidelines. The samples included 15 articles published between 1998 and 2019. From a total of 456 patients who underwent gonadectomy or gonadal biopsy, $6.14 \%$ had a premalignant lesion and most were postpubertal (82.14\%). A malignant lesion was found in $1.3 \%$ and all were postpubertal. Because the risk of malignancy is very low in prepubertal patients with CAIS, gonadectomy may be delayed until puberty is complete, allowing it to progress naturally; however, close follow-up of the patient is required.

Keywords: Androgen insensitivity syndrome, Gonadectomy, Neoplasia, Puberty

\section{Highlights}

Complete androgen insensitivity syndrome (CAIS) is a rare disorder of 46,XY karyotype with female external genitalia. In patients with CAIS, the risk of gonadal tumor increases with age and is estimated by $3.6 \%$ to $33 \%$. However, in our systematic review of 15 studies including 456 patients, the malignancy was detected only $1.3 \%$ and after 12 years of age. Maintenance of the gonads in CAIS may optimize the growth and acquisition of bone mass. Gonadectomy can lead to worsening of sexual function and high level of psychological stress in CAIS. Thus, we recommend gonadectomy might be delayed until puberty is complete.

\section{Introduction}

Androgen insensitivity syndrome (AIS) (OMIM \#300068) is an X-linked recessive disorder of sex development (DSD) in which androgen resistance is due to mutations in the androgen receptor $(A R)$ gene (OMIM *313700), located on Xq12. Complete AIS (CAIS) has a frequency of approximately 1:20,000 male births. CAIS is characterized by 46,XY karyotype, female external genitalia, testes located in the abdomen, inguinal ring or labioscrotal region, and internal genitalia with a blind ending vagina, absence of uterus and fallopian tubes, and no Wolffian development. ${ }^{1)}$ When the testes are preserved, puberty occurs spontaneously with normal breast development and female body contours, which are due to gonadal estrogen production and peripheral aromatization of testosterone, and primary amenorrhea due to the absence of uterus; lack of androgen action leads to absence or significant reduction of acne as well as pubic and axillary hair. ${ }^{2)}$

The AR is expressed in Sertoli and Leydig cells and in the peritubular compartments.

\author{
Accepted: 13 November, 2020 \\ Address for correspondence: \\ Beatriz Amstalden Barros \\ Rua Tessalia Vieira de Camargo, 126 \\ Cidade Universitaria, Campinas (SP) \\ 13083-887, Brazil \\ Email: b059191@dac.unicamp.br \\ https://orcid.org/0000-0002-7088- \\ 9058
}

Received: 30 July, 2020 
Defects in the AR may lead to changes in all these cell lines. Histological evaluation of 44 patients with CAIS undergoing prophylactic gonadectomy showed rapid loss of germ cells from the second year of life, Sertoli cell nodules in $24 \%$ of patients older than 7 years of age, reduced diameter of seminiferous tubules from 14 years of age, and interstitial fibrosis in most cases. ${ }^{3)}$ Other findings in the present study were atypical germ cells (enlarged nuclei, with irregularity or agglutination of the chromatin) in $1 / 3$ of the patients and dysgenetic seminiferous tubules in $18 \%$ of patients over 12 years of age. ${ }^{3)}$

The risk of gonadal tumor increases with age in patients with CAIS and is estimated at $3.6 \%$ at 25 years and $33 \%$ at 50 years. ${ }^{4}$ Germ cell tumors and their precursor lesion, the intratubular germ cell neoplasia or carcinoma in situ, may also be observed; both terms were recently replaced by germ cell neoplasia in situ (GCNIS) by the World Health Organization. ${ }^{5)}$ Tumors of nongerminal origin can also be found, including Leydig cell tumor, Sertoli cell tumor, sarcoma, and lymphoma. ${ }^{3)}$

Immunohistochemical markers help diagnose germ cell tumors in patients with DSD. Placental alkaline phosphatase (PLAP) and c-KIT (a receptor tyrosine kinase), are expressed in neoplastic in situ lesions such as gonadoblastoma and GCNIS. In addition, expression of octamer-binding transcription factor 3/4 (OCT3/4 or POU5F), a transcription factor of other genes, may be observed ${ }^{4)}$; abnormal and prolonged expression of this marker after birth and increased TSPY expression in germ cells determine the oncogenic potential of these tumors. ${ }^{3,46}$

Currently, indicating invasive procedures in patients with DSD is an important issue. ${ }^{4,5,7)}$ Patients and support groups cite the negative effects of gonadectomy and the consequences of non-physiological hormone replacement. ${ }^{8,9)}$ In patients with CAIS, peripheral conversion of testosterone to estrogen and intratesticular estrogen production allow development of spontaneous pubertal features. ${ }^{8,9)}$ Due to the rarity of CAIS, most follow-up studies included only a small number of patients from each modality. In the present systematic review, the frequency of malignancy in the testes of patients with CAIS based on age at gonadectomy or gonadal biopsy was determined to provide safety data regarding postponing gonadectomy until the end of puberty.

\section{Methods}

This systematic review was conducted according to the Preferred Reporting Items for Systematic Review and MetaAnalysis Protocols (PRISMA-P) guidelines. ${ }^{10)}$

In December 2019, a review of articles related to CAIS and the risk of gonadal malignancies was performed independently and simultaneously by the authors (BAB, LRO, and CRCS) using the PubMed, Ovid database, and Scopus databases and searching for the following terms: "Complete androgen insensitivity syndrome," "malignancy" AND "gonadectomy."

In the first selection stage, the titles and abstracts of all articles published from January 1990 to December 2019 were read and duplicated articles were excluded. The following inclusion criteria were applied: original articles in which patients with CAIS received gonadectomy or gonadal biopsy; availability of histopathological analysis of the gonads; information on the age at the time of the procedure. Letters to the editor, editorials, book chapters, review articles, case reports, and articles involving other aspects associated with CAIS or addressing individuals with partial androgen insensitivity syndrome (PAIS) were excluded, as well as articles written in a language unknown to the authors. The selected articles were carefully read by the authors and the following data were collected: title, authors, place of study, date of publication, number of patients with CAIS who received gonadectomy or gonadal biopsy, number of patients older and younger than 12 years of age with premalignant and malignant lesions, expression of immunohistochemical markers of malignancy, and study conclusion.

In the second phase, articles without access to the complete text were excluded. At all stages of data selection and extraction, disagreements between researchers were solved through discussion and consensus.

\section{Results}

The initial search with the keywords resulted in 192 articles (PubMed, 61; Ovid database, 99; Scopus, 32). In the first selection stage, 63 duplicated articles were excluded. After reading the remaining 129 titles and abstracts, 111 were excluded: 7 studies were in other text format ( 5 book chapters, 1 editorial, and 1 letter), 2 articles were in Bulgarian language. In addition, there were 31 reviews, 34 case reports, 32 articles investigating other aspects of patients with CAIS or related only to PAIS, and 5 articles published before 1990. The remaining 18 articles were selected for full reading.

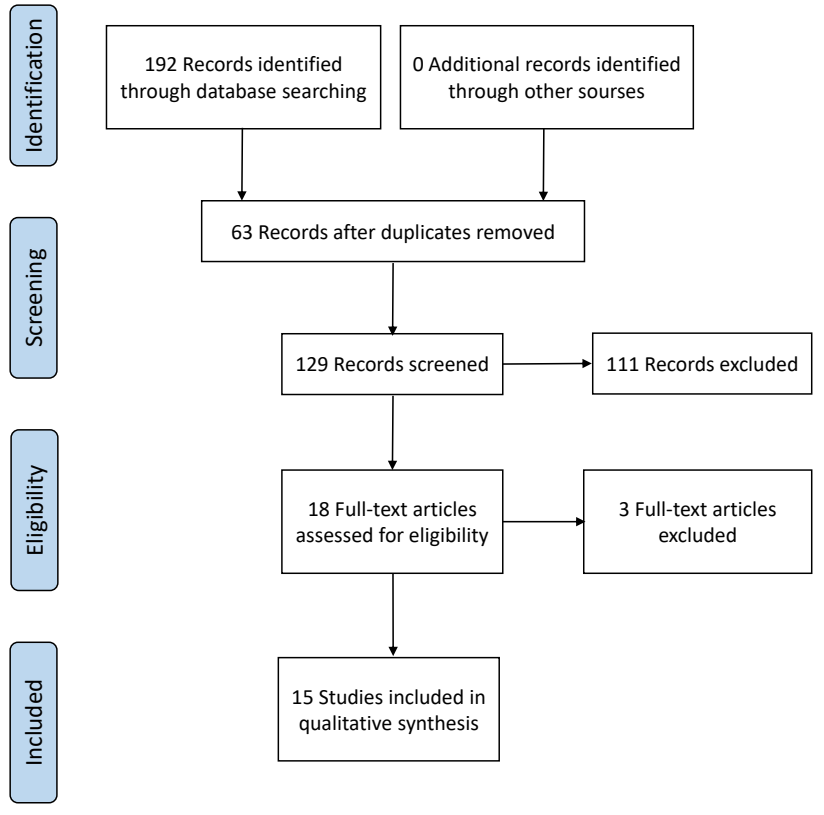

Fig. 1. PRISMA-P (Preferred Reporting Items for Systematic Review and MetaAnalysis Protocols) flow chart for data extraction. 
In the second selection stage, 3 studies were excluded: 2 because the full text could not be accessed and the other due to lack of information on patient age at gonadectomy. The remaining 15 articles were included for data extraction. The selection process of the articles is illustrated in Fig. 1 with a flowchart as recommended by PRISMA-P guidelines. ${ }^{10)}$

The articles included in the review were published from 1998-2017. Data on gonadal biopsy or gonadectomy based on patient age at the procedure are presented in Table 1.,5,11-23)

Among the 15 studies analyzed, malignant lesions at gonadectomy were reported in 2 studies: Chaudhry et al. ${ }^{5)}$ described a 30-year-old patient with seminoma and a 68-yearold woman with primary amenorrhea and malignant sex cord stromal tumor. Huang et al. ${ }^{23)}$ found 4 patients with seminoma, all older than 20 years. In 6 articles, premalignant lesions (GCNIS, Sertoli cell tumor, and gonadoblastoma) were detected, including in 5 children under 12 years of age and 23 girls older than 12 years. A total of 456 CAIS patients who underwent gonadectomy or gonadal biopsy were collected; $6.14 \%$ presented with a premalignant lesion at histological evaluation (82\% older than 12 years of age) and $1.31 \%$ presented with a malignant lesion, all older than 12 years (Table 1).

In 5 studies, immunohistochemical analysis of tumor markers was performed on the gonads (Table 2). ${ }^{3,5,14,15,19)}$ The markers used included PLAP, CD117 or C-KIT, OCT3/4, activator protein $2_{\gamma}$ nuclear $\left(\mathrm{AP} 2_{\gamma}\right)$, vimentin and/or KI67. Hannema et al. ${ }^{3)}$ evaluated PLAP and AP2y markers in 23 gonadectomies and found 6 subjects were positive for both ( 4 children under 12 years of age and 2 older than 12 years). Notably, in prepubertal cases, the markers were found more diffusely; however, in 2 patients older than 12 years of age, most of the testes were negative but foci of seminiferous tubules contained many positive germ cells. One patient was 18 years of age and had a GCNIS diagnosis and another was 53 years of age with some atypical germ cells but did not show classical GCNIS pattern. ${ }^{3)}$ Cools et al. ${ }^{14)}$ evaluated gonads from 15 patients for PLAP, CD117, and OCT3/4, and found positivity for all markers in patients younger than 9 months of age and in a 18-year-old patient with a GCNIS. Wünsch et al. tested for PLAP, CD117, and KI67 in 7 patients and all were negative. ${ }^{19)}$ Chaudhry et al. ${ }^{5)}$ performed immunohistochemical analysis in most cases and found 9 subjects were suspected with premalignant lesions, 6 were positive for PLAP, 3 for CD117, 1 for OCT3/4, and a 68-year-old patient with a malignant sex cord stromal tumor was positive for vimentin. Regarding the PLAP-positive cases, 2 were younger than 12 years of age and had a GCNIS in which 1

Table 1. Number of premalignant or malignant lesion in patients with complete androgen insensitivity syndrome according to age of gonadectomy or gonadal biopsy

\begin{tabular}{|c|c|c|c|c|c|c|}
\hline Study & No. & $\operatorname{Age}^{*}(\mathrm{yr})$ & $\mathrm{PML}<12 \mathrm{yr}$ & $\mathrm{PML}>12 \mathrm{yr}$ & $\mathrm{ML}<12 \mathrm{yr}$ & $M L>12 y r$ \\
\hline Kriplani et al., ${ }^{11)}$ (1998) & 2 & 21.5 & 0 & 0 & 0 & 0 \\
\hline Ahmed et al., ${ }^{12)}(2000)$ & 65 & - & 0 & 0 & 0 & 0 \\
\hline Yalinkaya and Yayla ${ }^{13)}$ (2003) & 2 & 20 & 0 & 0 & 0 & 0 \\
\hline Cools et al., ${ }^{14)}(2005)$ & 15 & - & 0 & 1 & 0 & 0 \\
\hline Hannema et al., (2006) & 44 & 5.5 & 0 & 2 & 0 & 0 \\
\hline Cheikhelar et al., ${ }^{15)}$ (2008) & 25 & 12.5 & 0 & 1 & 0 & 0 \\
\hline Purves et al.. ${ }^{16)}$ (2008) & 29 & 18.2 & 0 & 0 & 0 & 0 \\
\hline Kravarusic et al.., ${ }^{17)}$ (2011) & 6 & 12.6 & 0 & 0 & 0 & 0 \\
\hline Dobanovacki et al., ${ }^{18)}$ (2012) & 4 & 6.3 & 0 & 0 & 0 & 0 \\
\hline Wünsch et al. ${ }^{19)}$ (2012) & 7 & 15.7 & 0 & 0 & 0 & 0 \\
\hline Esposito et al., ${ }^{20)}(2015)$ & 10 & 11.5 & 0 & 0 & 0 & 0 \\
\hline Liu et al., ${ }^{21)}(2014)$ & 30 & 22.3 & 0 & 9 & 0 & 0 \\
\hline Wang et al., ${ }^{22)}$ (2014) & 5 & 20.4 & 0 & 0 & 0 & 0 \\
\hline Huang et al., ${ }^{23)}$ (2017) & 79 & 19 & 3 & 5 & 0 & 4 \\
\hline Chaudhry et al.,. (2017) & 133 & 14 & 2 & 5 & 0 & 2 \\
\hline Total & 456 & & 5 & 23 & 0 & 6 \\
\hline
\end{tabular}

PML, premalignant lesion; ML, malignant lesion; -, not informed.

*Age at gonadectomy or gonadal biopsy (average in years).

Table 2. Evaluation of immunohistochemical tumor markers in gonadal biopsy or gonadectomy in patients with complete androgen insensitivity syndrome

\begin{tabular}{|c|c|c|c|}
\hline Study & Markers studied & No. & Positive Markers \\
\hline Cools et al., ${ }^{14)}(2005)$ & PLAP, OCT3/4, CD117, & 15 & PLAP, OCT3/4, CD 117: 1 (>12 yr) \\
\hline Hannema et al., ${ }^{3)}(2006)$ & PLAP, $A P 2_{\gamma}$ & 23 & PLAP, AP2r: $6(4<12 y r)$ \\
\hline Cheikhelar et al., ${ }^{15)}$ (2008) & PLAP, CD 117 & 1 & PLAP, CD 117: 1 (>12 yr) \\
\hline Wünsch et al., ${ }^{19)}$ (2012) & PLAP, CD 117, KI 67 & 7 & 0 \\
\hline Chaudhry et al., ${ }^{5}$ (2017) & PLAP, CD 117, OCT3/4, vimentin & 9 & PLAP: 6 ( $2<12 \mathrm{yr})$; CD 117: 3 (2<12 yr); OCT3/4: 1 (1<12 yr); Vimentin: 1 (>12 yr) \\
\hline
\end{tabular}

* Number of patients submitted to immunohistochemical staining. 
case also had a maturation delay of germ cells and 4 were older than 12 years ( 3 cases of GCNIS and 1 case of seminoma). ${ }^{5}$

Although the use of tumor markers varied in each study and immunohistochemical analysis was only performed in some studies when the histology was suspicious for malignancy, the results showed that tumor markers can be found in young patients and immunohistochemical analysis should be performed whenever patients receive gonadectomy or gonadal biopsy.

\section{Discussion}

In this systematic review of 15 studies, the gonadal aspect of a large number of patients with CAIS was evaluated. When evaluating the results of histological analysis based on age from a sample of 456 patients, a low rate of malignancy was detected (6.14\% of premalignant lesions, the majority (81.4\%) after 12 years of age, and $1.3 \%$ of malignancies, all in adult women).

Maintenance of the gonads in patients with CAIS may optimize growth and acquisition of bone mass. ${ }^{15)}$ Patients with preserved gonads exhibit pubertal stretch at the same age as nonaffected individuals, showing that estrogens can stimulate growth rate in the absence of androgens. In fact, estrogens play an important role in modulating linear growth by action on the GH-IGF1 axis as well as on the growth plate. ${ }^{24,25)}$ In addition, other hormones secreted by the testes, such as INSL3, act directly on osteoblastic function and bone mineralization. ${ }^{26)}$ However, women with CAIS have lower bone mass peak than unaffected individuals, regardless of endogenous or exogenous estrogen source, probably due to the lack of direct androgen action on the bone. ${ }^{8,27)}$

Another important factor to consider is the psychological effects of gonadectomy, however, this issue was addressed in only a few studies. Gonadectomy can lead to worsening of sexual function and high level of psychological stress in women with CAIS ${ }^{8)}$ Furthermore, in a study with 14 adult patients between 20 and 60 years of age (mean, 48 years), in which the relationship between psychosocial status and therapeutic approaches was evaluated, the majority of subjects (78\%) considered the ideal time for gonadectomy or vaginoplasty would be after puberty or adulthood. ${ }^{28)}$

However, the incidence of germ cell tumors is higher in patients with CAIS than in the general population, especially in adulthood. Due to the higher frequency of gonadal morphology variations, the diagnosis of premalignant lesions may be difficult. Whenever possible, analyzing immunohistochemical markers such as PLAP, AP2 $2_{\gamma}, \mathrm{c}-\mathrm{KIT}$, and OCT3/4 through gonadal biopsy is important because their presence may indicate risk of malignancy. These markers are useful for detecting GCNIS in children with DSD, however, expression in dysgenetic gonads and in normal gonads of infants may also be increased. ${ }^{5,29)}$ Patients younger than 2 years of age had higher positivity for PLAP, AP2 $2_{\gamma}, \mathrm{c}-\mathrm{KIT}$, and OCT3/4, as well as patients with premalignant lesions. The presence of OCT3/4, despite being associated with higher risk of malignancy and present in cases of GCNIS, gonadoblastomas, and seminomas, does not differentiate simple delay of germ cell maturation from premalignant lesions in very young children ${ }^{30)}$ Consequently, these markers should not be used for detection of GCNIS at this age.

The current consensus in the management of patients with DSD indicates the guidelines should be based on the holistic well-being of the child and the future adult, aiming at minimizing physical and psychosocial risks, preserving potential fertility, respecting the individual's right to participate in the decisions that will affect them, and avoid irreversible treatments not essential for their health. ${ }^{9)}$ In addition, seeking psychosocial support that promotes the healthy development of sexuality and gender identity is important to ensure decisions regarding the timing of gonadectomy and the beginning of hormone replacement are considered together with the patient and family."

Considering the low rate of malignancy in CAIS patients and advantages of preserving gonads, patients with CAIS should be monitored with frequent imaging studies of the testes and gonadal biopsy with immunohistochemistry when necessary until puberty. Subsequently, the decision regarding gonadectomy can be made. The possibility of spontaneous puberty with physiological development of secondary sexual characteristics, stimulation of stature growth and bone maturation, and minimization of the psychological effects of treatment are relevant positive aspects of postponing the definitive treatment in these patients. The conservative management of the gonads in CAIS is also a recommendation of the Consensus on the management of $\mathrm{DSD}^{9)}$ and according to recent European research, gonadectomy is recommended in early adulthood in $67 \%$ of centers that use the I-DSD Registry data. ${ }^{27)}$

\section{Conflicts of interest}

No potential conflict of interest relevant to this article was reported.

\section{References}

1. Oakes MB, Eyvazzadeh AD, Quint E, Smith YR. Complete androgen insensitivity syndrome - a review. J Pediatr Adolesc Gynecol 2008;21:305-10.

2. Mongan NP, Tadokoro-Cuccaro R, Bunch T, Hughes IA. Androgen Insensitivity Syndrome. Best Pract Res Clin Endocrinol Metab 2015;29:569-80.

3. Hannema SE, Scott IS, Rajpert-De Meyts E, Skakkebæk NE, Coleman N, Hughes IA. Testis development in the complete androgen insensitivity syndrome.J Pathol 2006;208:518-27.

4. Cools M, Drop SL, Wolffenbuttel KP, Oosterhuis JW, Looijenga LH. Germ cell tumors in the intersex gonad: old paths, new directions, moving frontiers. Endocr Rev 2006;27:468-84.

5. Chaudhry S, Tadokoro-Cuccaro R, Hannema SE, Acerini CL, Hughes IA. Frequency of gonadal tumours in complete 
androgen insensitivity syndrome (CAIS): a retrospective case-series analysis. J Pediatr Urol 2017;13:498.

6. Gidekel S, Pizov G, Bergman Y, Pikarsky E. Oct-3/4 is a dose-dependent oncogenic fate determinant. Cancer Cell 2003;4:361-70.

7. Deans R, Creighton SM, Liao LM, Conway GS. Timing of gonadectomy in adult women with complete androgen insensitivity syndrome (CAIS): patient preferences and clinical evidences. Clin Endocrinol 2012;76:894-8.

8. Döhnert U, Wünsch L, Hiort O. Gonadectomy in complete androgen insensitivity syndrome: why and when? Sex Dev 2017;11:171-4.

9. Lee PA, Nordenström A, Houk CP, Ahmed SF, Auchus R, Baratz A, et al. Global disorders of sex development update since 2006: perceptions, approach and care. Horm Res Paediatr 2016;85:158-80.

10. Moher D, Liberati A, Tetzlaff J, Altman DG, The PRISMA Group. Preferred Reporting Items for Systematic Reviews and Meta-Analyses: the PRISMA statement PLoS Med 2009;6:e1000097.

11. Kriplani A, Abbi M, Ammini AC, Kriplani AK, Kucheria K, Takkar K. Laparoscopic gonadectomy in male pseudohermaphrodites. Eur J Obstet Gynecol Reprod Biol 1998;81:37-41.

12. Ahmed SF, Cheng A, Dovey L, Hawkins JR, Martin H, Rowland J, et al. Phenotypic features, androgen receptor binding, and mutational analysis in 278 clinical cases reported as androgen insensitivity syndrome. J Clin Endocrinol Metab 2000:85:658-65.

13. Yalinkaya A, Yayla M. Laparoscopy-assisted transinguinal extracorporeal gonadectomy in six patients with Androgen Insensitivity Syndrome. Fertil Steril 2003;80:429-33.

14. Cools M, van Aerde K, Kersemaekers AMF, Boter M, Drop SL, Wolffenbuttel KP, et al. Morphological and immunohistochemical differences between gonadal maturation delay and early germ cell neoplasia in patients with undervirilization syndromes. J Clin Endocrinol Metab 2005;90:5295-303.

15. Cheikhelard A, Morel Y, Thibaud E, Lortat-Jacob S, Jaubert F, Polak M, et al. Long-term follow-up and comparison between genotype and phenotype in 29 cases of Complete Androgen Insensitivity Syndrome. J Urol 2008;180:1496501.

16. Purves JT, Miles-Thomas J, Migeon C, Gearhart JP. Complete androgen insensitivity: the role of the surgeon. J Urol 2008; 180:1716-9.

17. Kravarusic D, Seguier-Lipszyc E, Feigin E, Nimri R, Nagelberg N, Freud E. Androgen insensitivity syndrome: risk of malignancy and timing of surgery in a paediatric and adolescent population. Afr J Paediatr Surg 2011;8:1948 .

18. Dobanovacki D, Jokic RR, Vuckovic N, Privrodski JDJ,
Katanic DJ, Tatic MR, et al. Complete androgen insensitivity syndrome: review of four cases. Cent Eur J Med 2012;7:72932.

19. Wünsch L, Holterhus PM, Wessel L, Hiort O. Patients with disorders of sex development (DSD) at risk of gonadal tumour development: management based on laparoscopic biopsy and molecular diagnosis. BJU Int 2012;110:E958-65.

20. Esposito C, Escolino M, Bagnara V, Eckoldt-Wolke F, Baglaj M, Saxena A, et al. Risk of malignancy and need of surgery in pediatric patients with Morris or Y-chromosome Turner: a multicenter survey. J Pediatr Adolesc Gynecol 2015;28:333-6

21. Liu AX, Shi HY, Cai ZJ, Liu A, Zhang D, Huang HF, et al. Increased risk of gonadal malignancy and prophylactic gonadectomy: a study of 102 phenotypic female patients with Y chromosome or Y-derived sequences. Hum Reprod 2014;29:1413-9.

22. Wang Z, Sa YL, Ye XX, Zhang J, Xu YM. Complete Androgen Insensitivity Syndrome in juveniles and adults with female phenotypes. J Obstet Gynaecol Res 2014;40:2044-50.

23. Huang, H, Wang, C, Tian Q. Gonadal tumour risk in 292 phenotypic female patients with Disorders of Sex Development containing Y chromosome or Y-derived sequence. Clin Endocrinol 2017;86:621-7.

24. Countant R, de Casson FB, Rouleau S, Douay O, Mathieu E, Gatelais F, et al. Divergent effect of endogenous and exogenous sex steroids on the insulin like growth factor response to growth hormone in short normal adolescents. J Clin Endocrinol Metab 2004;89:6185-92.

25. Perry RJ, Farquharson T, Ahmed SF. The role of sex steroids in controlling pubertal growth. Clin Endocrinol 2008;68:415 .

26. Ferlin A, Selice R, Carraro U, Foresta C. Testicular function and bone metabolism - beyond testosterone. Nat Rev Endocrinol 2013;9:548-54.

27. Tack LJW, Maris E, Looijenga LHJ, Hannema SE, Audi L, Köhler B, et al. Management of gonads in adults with androgen insensitivity: an International survey. Horm Res Paediatr 2018;90:236-46.

28. Wisniewski AB, Migeon CJ, Meyer-Bahlburg HFL, Gearhart JP, Berkovitz GD, Brown TR, et al. Complete androgen insensitivity syndrome: long-term medical, surgical, and psychosexual outcome. J Clin Endocrinol Metab 2000;85:2664-9.

29. Slowikowska-Hilczer J, Romer TE, Kula K. Neoplastic potential of germ cells in relation to disturbances of gonadal organogenesis and changes in karyotype. J Androl 2013;24:270-8.

30. Jones TD, Ulbright TM, Eble JN, Baldridge LA, Cheng L. OCT4 staining in testicular tumors: a sensitive and specific marker for seminoma and embryonal carcinoma. Am J Surg Pathol 2004;28:935-40. 